\title{
Problem-adapted Modeling of RF MEMS Switches
}

\author{
Gabriele Schrag, Thomas Künzig, Martin Nießner \\ Institute for Physics of Electrotechnology, Munich University of Technology, \\ Arcisstr. 21, D-80290 Munich, Germany \\ schrag@tep.ei.tum.de
}

\begin{abstract}
We present two problem-adapted, tailored modeling approaches for RF-MEMS switches, which are applicable, in principle, to any capacitive MEMS device and are based on finite element analysis (FEA) combined with analytical, lumped-element and/or reduced-order modeling techniques. The first model has been tailored for detailed investigations on device-level (problem-adapted FE model), while the second approach is suited for system-level simulation and based on macromodels generated from a discretized FE model of the device. Both approaches enable efficient and physics-based simulation of static and dynamic device characteristics, which is demonstrated by comparing the simulation results of two different RF MEMS switch designs to optical measurements.
\end{abstract}

Key words: RF MEMS switch, finite element modeling, system-level modeling, problem-adapted and tailored modeling, model validation

\section{Introduction and Motivation}

In general, finite element analysis (FEA) is the method of choice for detailed investigations of MEMS devices. However, in the case of complex device geometries and several involved energy domains this method often entails unacceptably long simulation times, weak convergence or even becomes prohibitive. The derivation of tailored, timeefficient but, at the same time, physics-based and predictive (i.e. scalable) models is therefore a major issue in the design of microsystems. In this work, two different approaches meeting these demands will be exemplified for two RF MEMS switch variants.

\section{Problem Description - Efficient Models for RF MEMS Switch Design}

The operation principle of the considered switches is schematically shown in Fig. 1.

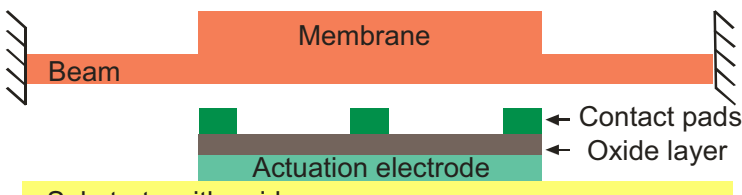

Substrate with oxide

Fig. 1. Operation principle of the electrostatically actuatable MEMS switches considered in this work.

Both variants have been fabricated by [1] and consist of a gold membrane suspended by four beams, which can be electrostatically pulled down towards several contact pads in order to close an RF signal line. Switch \#1 is designed with a sliced and switch \#2 with a perforated membrane, which can be seen in Figs. 2 and 3, respectively. A proper model of both designs has to take into account at least three different energy domains: mechanical domain, electrical domain, and viscous damping.

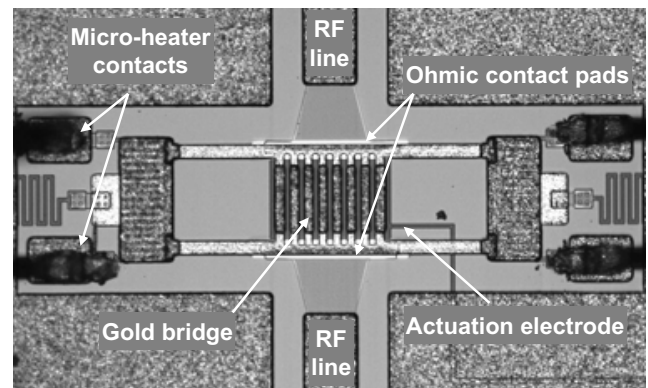

Fig. 2. Microphotograph of switch \#1: meandershaped, resistive heaters are integrated underneath both anchors intended to counteract stiction by exerting shear forces on the bridge.

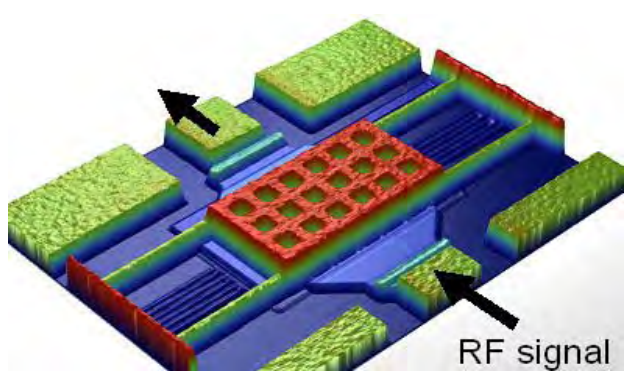

Fig. 3. Topographic view of RF MEMS switch \#2 obtained by white light interferometry. The perforated membrane can be actuated electrostatically in order to close an RF signal line. 
Additionally, switch \#1 is equipped with resistive heaters underneath the anchors in order to reactivate it in case of stiction-induced failure by heating up the bridge and exerting shear forces to it. In this case, also the coupling to the thermal domain has to be included in the model. In the following, we will derive FE-based models that are strongly adapted and tailored to the given optimization task.

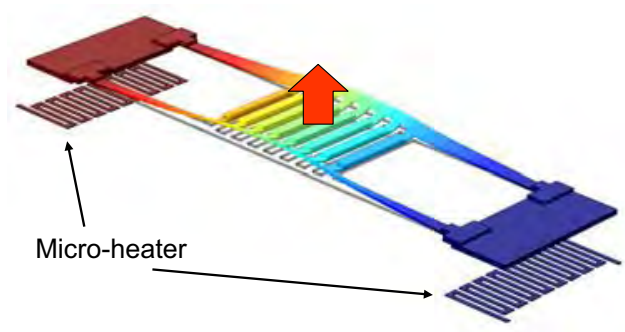

Fig. 4: Schematic view of the embedded recovery capability (switch \#1). Resistive micro-heaters underneath each anchor enable heating up the bridge in order to recover it from the failure mode.

As shown in Fig. 4, switch \#1 is equipped with a novel thermal recovery mechanism [2] to counteract stiction caused by microwelding [3] and/or dielectric charging [4], two phenomena which significantly impair the reliability of such devices. Hence, a model is required that is detailed enough to deliver insight into the device operation (internal heat propagation, e.g.) but, on the other hand, fast enough to allow for comprehensive design and optimization studies.

The model of switch \#2, however, is intended for investigations on the switching dynamics in context with system considerations. Thus, very fast (means reduced number of degrees of freedom), but yet physics-based, accurate models suited for system simulation are needed in this case.

\section{FE-Based Problem-adapted Model of Switch \#1}

\subsection{Modeling}

For switch \#1, a detailed (3D), coupled, semianalytical FE-model has been derived, where the mechanical, the thermal, and the electrostatic energy domains and their respective couplings have been considered. In order to make the problem tractable and to keep the simulation expense in an acceptable range, the Joule heating and the electrostatic actuation have been taken into account by introducing (semi-) analytical models, while the thermo-mechanical part is modeled by a coupled FEA. This approach is outlined in Fig. 5 and described in detail in [5].

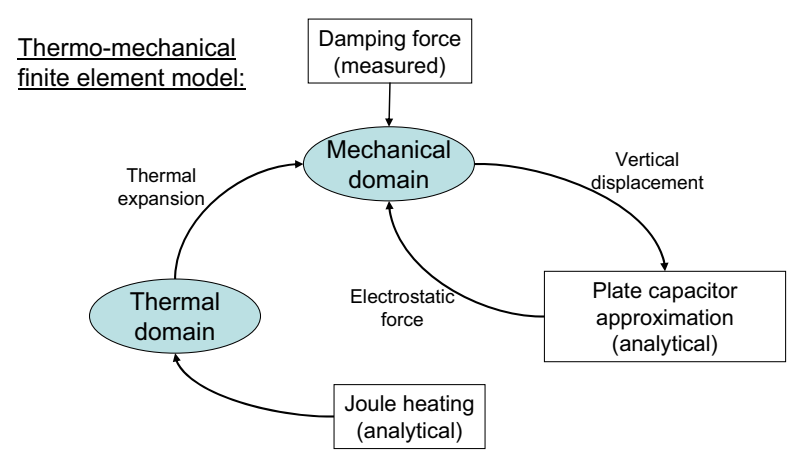

Fig. 5. Outline of thermo-mechanical FE model of switch \#1. Electrical und fluidic effects as well as the Joule heating are included in the finite element model as (semi-)analytical models.

All model parameters have been properly calibrated and validated by white-light interferometer and Laser Doppler vibrometer measurements. E.g., initial stress inside the movable membrane has been extracted from the fundamental eigenfrequency and included in the mechanical model. Fluidic damping has been determined by measuring damped oscillations of the switch membrane under normal pressure conditions and, subsequently, considered by introducing a constant damping factor. The gap underneath the gold membrane has been extracted by optically measuring the displacement of the membrane in the quasistatic pull-in characteristics.

The calibrated and validated model can now be used to evaluate the restoring mechanism in the failure scenario, viz. the temperature distribution and the thermally induced forces exerted on a potential sticking point. To this end, the sequential simulation procedure depicted in Fig. 6 has been developed. A pure thermal FE analysis of the device including also the substrate (step 1) delivers the temporal evolution of the temperature distribution. Step 2 comprises first a pure mechanical contact simulation (2a) to calculate the deformation of the switch in its closed position applying an electrostatic force. Then the restoring mechanical force on a fixed contact point is extracted (2b). Finally, the transient temperature distribution extracted from step 1 enters the thermo-mechanical model (step 2c) as boundary condition and the total force acting on the welded contact is extracted. Subtracting the mechanical restoring force (obtained by step (2b)) from the so-determined value of the total force delivers the force exerted solely by the thermal recovery mechanism, and, thus, an estimate for its effectiveness during a potential failure situation. 

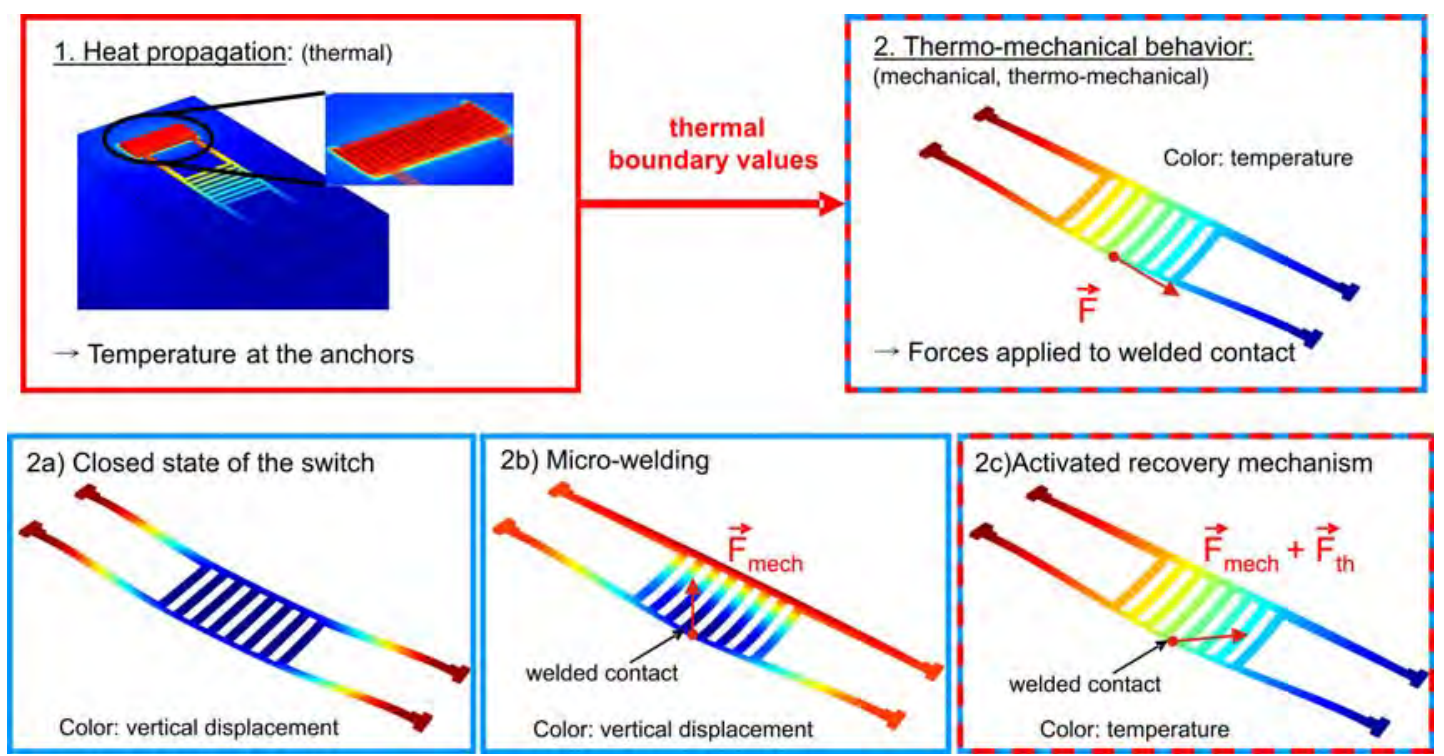

Fig. 6. Overview of the sequential simulation procedure applied to extract the thermally induced forces on potential sticking points. The colors of the boxes refer to the respective type of simulation (red: thermal, blue: mechanical, red-blue: thermo-mechanical).

\subsection{Results - Evaluating the Efficiency of the Thermal Recovery Mechanism}

During the model derivation it revealed, that special diligence has to be set on the model calibration and validation in order to ensure accurate and predictive simulation results. Since the heat propagation inside the device is a crucial parameter for the effectiveness of the recovery mechanism and cannot be assessed directly through measurements, the final thermo-electro-mechanical model has been verified by comparing the simulated thermally induced steady-state and transient deflections of the bridge to optically measured data (Figs. 7 and 8). The very good accordance between simulated and measured values illustrates the confidence level of the derived model for the following investigations, where important factors impacting the mechanism's functionality shall be identified and understood in order to estimate the potential for improvement and optimization of the device.

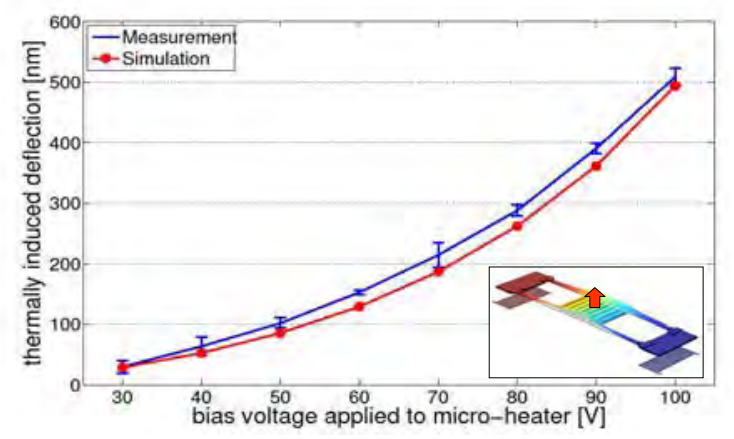

Fig. 7: Thermally induced vertical deflection of the bridge for different voltages applied to the heater.

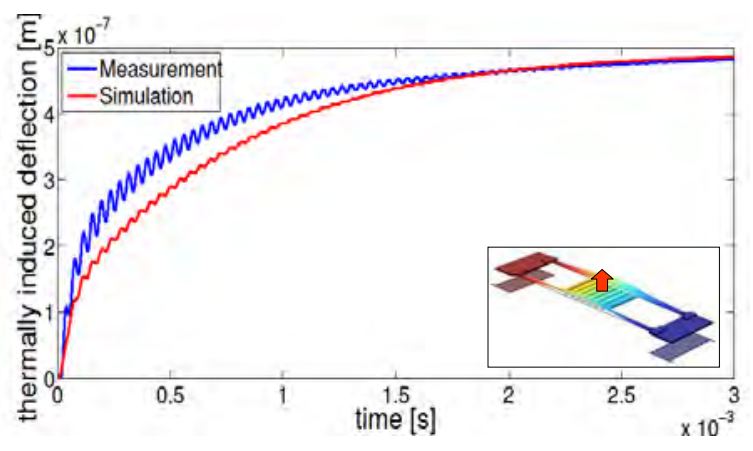

Fig. 8: Transient thermal expansion of the gold bridge caused by a heating voltage of $100 \mathrm{~V}$ and a current of $3.6 \mathrm{~mA}$. Both, simulation and measurement show a superimposed oscillation at the mechanical resonance frequency of the bridge.

One insight (among others), which has been gained with the help of the derived model, is the temperature distribution inside the device depicted in Fig. 9. It can be seen that the major temperature rise occurs inside the switch membrane and the hot anchor, but, on the other hand, the largest fraction of the entire heat is propagating into the substrate. This emphasizes the decisive role of the oxide layers between anchor and substrate as design parameter to tune the heating efficiency. As a consequence of this finding, the thermally induced forces increase proportionally with increasing thickness of the oxide layer. Another important measure for the evaluation of the recovery mechanism is the total force and, herewith, the amount of the thermally induced force and its variation along the gold membrane, which can be extracted from the simulations. 

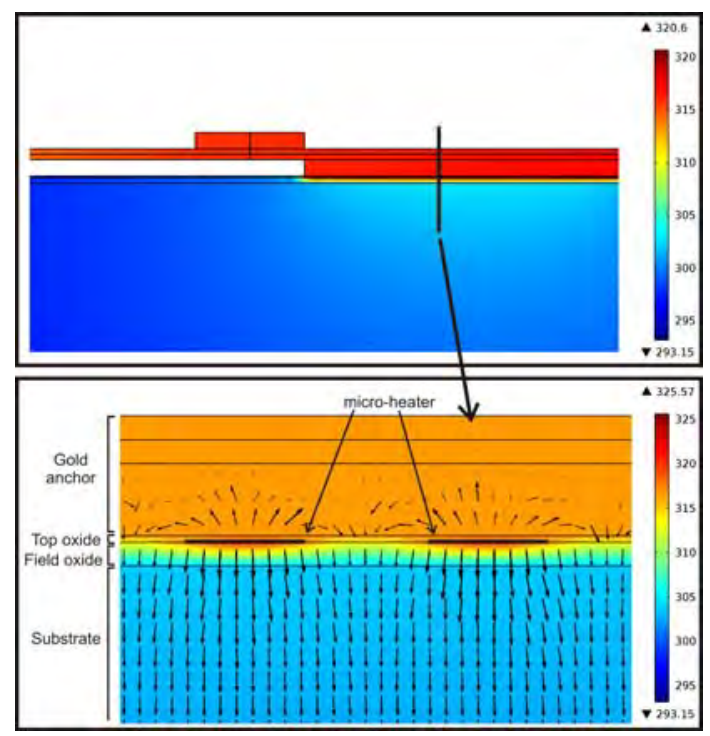

Fig. 9. Stationary temperature distribution of two cross-sections of the switch at the hot anchor: the black arrows illustrate the heat flux.

Fig. 10 shows - as expected - no dependence of the mechanical restoring force on the position, while the thermally-induced force is varying slightly along the bridge due to the fact that its value is correlated with the relative volume dilatation. However, the maximum variation is only of about $25 \%$, and, hence, shows no dramatic dependence. Additionally, the total value of the forces exerted on potential sticking points can be evaluated to lie between about $180 \mu \mathrm{N}$ to $220 \mu \mathrm{N}$. This value constitutes a promising result, since it is of the same order as those reported for welded gold contacts $(100 \mu \mathrm{N}-400 \mu \mathrm{N})$ [13], which proves, in principal, the basic functionality of the considered design.

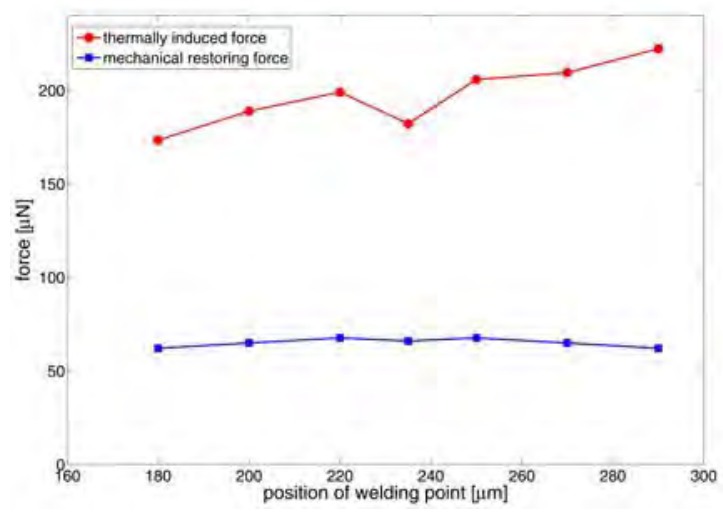

Fig. 10. Mechanical and thermally induced forces acting on a sticking point as a function of the distance from the hot anchor.

\section{System-level Model of Switch \#2}

\subsection{Modeling}

The model of switch \#2 is intended for investigating and optimizing the switching operation of the device. Consequently, the mechanical energy domain including mechanical contact, electrostatic actuation and damping induced by the surrounding air as well as their bidirectional couplings have to be properly taken into account. In contrast to switch \#1, modeling of the damping forces acting on the switch is more crucial here, since it essentially determines the switching operation (e.g., switching time, bouncing of the switch). Hence, it has to be included in detail on a proper physical and, preferably, scalable basis, which makes 3D, transient simulations on finite element level prohibitive due to the complex geometry (perforation holes inside the membrane). This is why we followed the hierarchical modeling scheme towards a system-level model depicted in [6,7]. The device is decomposed (Fig. 11) and submodels of each energy domain are formed using strictly flux-conserving reduced-order and/or compact modeling techniques within the framework of the generalized Kirchhoffian network theory.

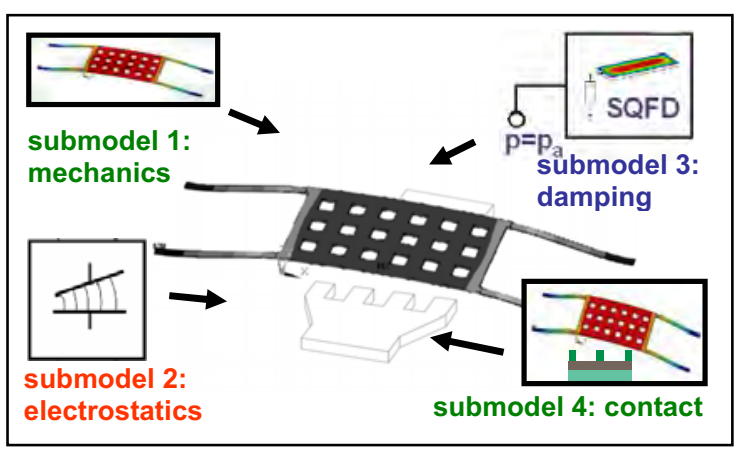

Fig. 11. Subdomain models to be derived for modeling of the switch on system level.

The basis of the mechanical submodel of the suspended membrane is the modal superposition technique described in $[8,9]$. The eigenmode shapes and frequencies of the suspended membrane are calculated in a FEM simulation tool. The most significant modes are identified and used to formulate a macromodel in terms of the modal amplitudes consisting of only one second-order differential equation per included eigenmode. Residual stress in the suspended membrane induced by the fabrication process has been taken into account by calibrating the fundamental eigenfrequency to the measured one.

The submodel of the electrostatic domain consists basically of a modified plate capacitor approach applied to the perforated membrane structure. The electrostatic forces are formulated in terms of the modal coordinates and transferred accordingly to the mechanical model. For details the reader is referred to [9]. 
The viscous damping forces are included by applying the mixed-level approach as presented in $[9,10]$. It is based on the Reynolds equation, which is evaluated by a fluidic Kirchhoffian network distributed over the device geometry. At perforations and outer boundaries lumped physics-based fluidic resistances are added accounting for the additional pressure drops at these locations (see Fig. 12). Consequently, this mixed-level model does not constitute a pure lumped element model and - depending on the granularity of the finite network - still exhibits a rather large number of degrees of freedom. However, the advantage of this approach is that it can be tailored to the topography of the real structure, i.e. take into account all perforations and - in the case of the considered switch - also locally varying gap heights which occur in our case due to the elevated contact pads and electrode fingers.

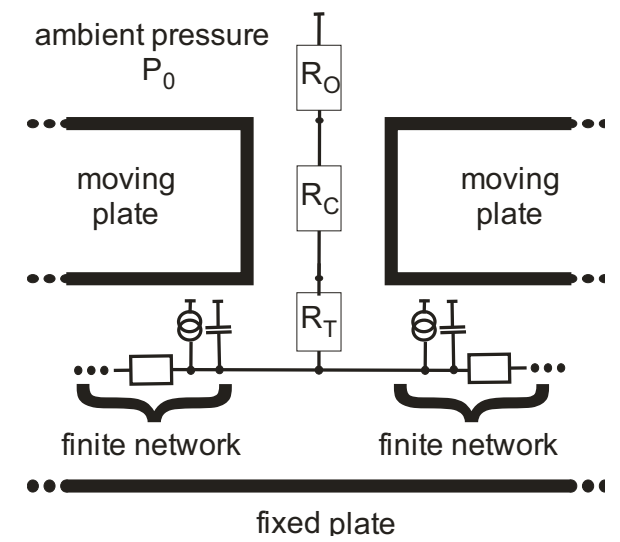

Fig.. 12: Mixed-level approach for modeling the viscous damping due to the surrounding air. Underneath the plate the Reynolds' equation is solved applying finite networks. The pressure drop due to the perforations is included by embedding the fluidic resistance $R_{T}$ for the region, where the fluid enters the channel, $R_{C}$ to model the channel resistance, and $R_{O}$ to model the orifice flow at the outlet.

The mechanical contact between membrane and contact pads has been taken into account by introducing contact forces at the respective locations. These have been formulated in terms of the modal coordinates comprising also higher-order modes. Hence, the resulting model enables also to emulate the bouncing of the membrane when hitting the counter electrode (Fig. 15). For details see [11].

All described submodels have been derived on the basis of detailed 3D FE simulations and have been incorporated in an on-purpose developed Matlab-based toolbox, which enables the derivation of system-level models starting from a discretized FE model of the device (Fig. 13). The so-derived model is finally translated into a hardware description language (Verilog-A, VHDL-AMS, e.g.) ending up in a physics-based and very efficient, energy-coupled reduced-order model that is formulated in terms of generalized Kirchhoffian networks and can be directly implemented in any commercial system-level simulator. The strength of this method is that all submodels, even the damping model of the perforated membrane, are fully parametrized and contain the physical dependencies accurately, while the model exhibits a reduced number of degrees of freedom, thus, enabling a fast and efficient simulation of the transient device behavior, even for complex geometries.

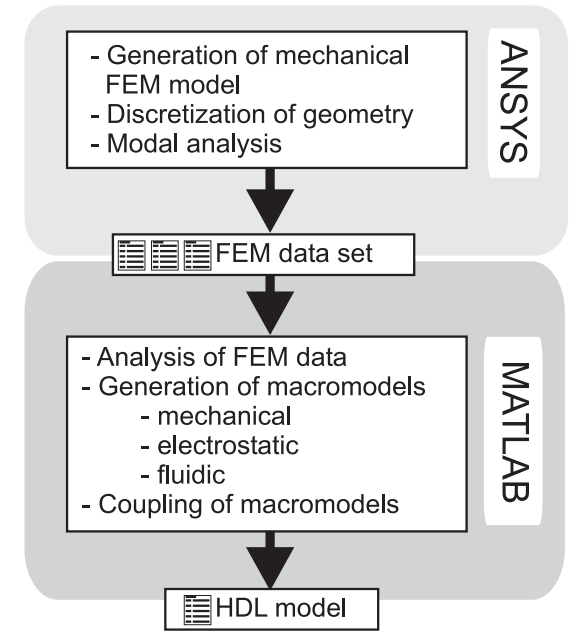

Fig.. 13: Workflow for the generation of system-level models from a FE discretization of the device applying an on-purpose developed Matlab toolbox.

\subsection{Results - Investigation of Switching Dynamics}

For the system-level model of switch \#2 the single submodels presented in section 3.2 have been extensively calibrated by FE simulations and measurements, so the modularly composed system-level model is supposed to deliver reliable and accurate results. This has been proven by comparing the simulated and measured response of the switch to a rectangular voltage waveform and recording the release to its respective rest position. The results in Fig. 14 show that the model correctly reproduces the electromechanical spring softening in the actuated state as well as the increased viscous damping due to the decreased air gap. This proves that the electromechanical coupling as well as the damping effects and their scaling with the device and ambient parameters have been properly taken into account, which is due to the physics-based modeling approach underlying the model derivation. The accuracy of the damping model alone has been investigated elsewhere and shows good results over a 
broad range of variations in geometrical dimensions and the ambient pressure [12].

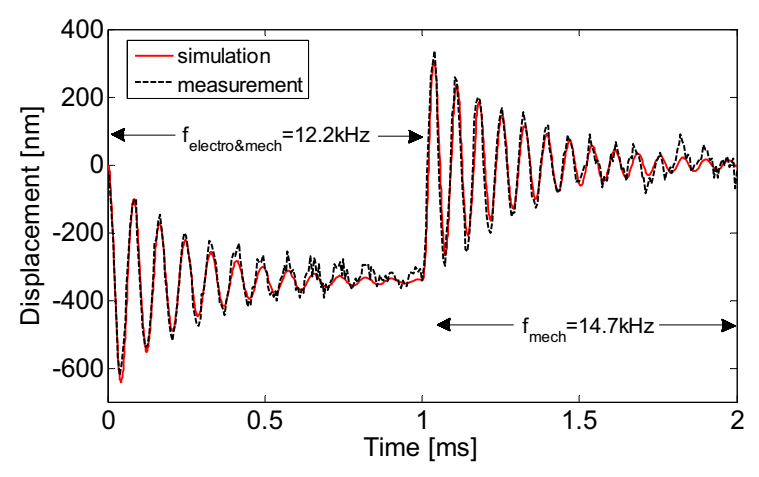

Fig. 14. Measured and simulated response of the membrane to a rectangular voltage waveform.

With the help of the so-validated model, the switching behavior can be studied (Fig. 15). The optically measured data shows that the model reproduces the closing of the switch accurately concerning switching time as well as the bouncing after hitting the contact. Thus, the model can be now used to optimize the switch with respect to the switching time, switching voltage and the contact pressure, also in conjunction with the surrounding system.

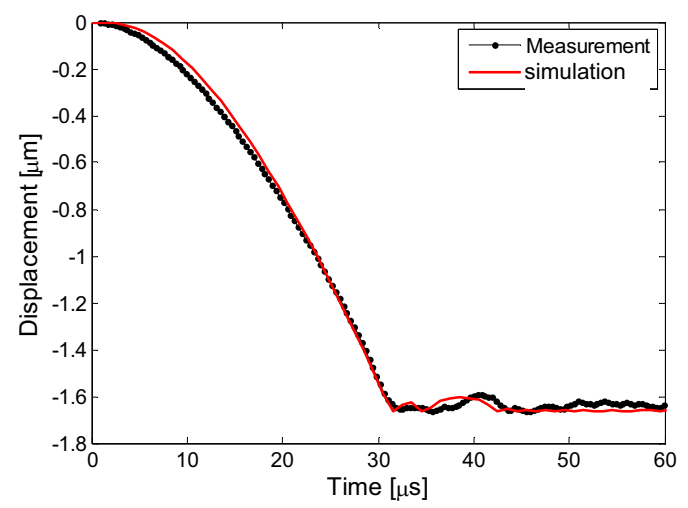

Fig. 15. Closing of the switch: comparison between optically measured and simulated displacement.

\section{Conclusions}

We presented two approaches to derive problem-adapted models for exemplary RF MEMS switch designs. These models have been derived on the basis of a general, physics-based philosophy, which is extendable to any other electro-mechanical-fluidic system. The focus in this work has been laid upon tailoring the respective models to the given specific needs and practicalities in order to obtain accurate results on a physical basis with an acceptable computational expense and to get models suited for predictive simulations within the design and optimization process.

\section{References}

[1] J. lannacci, F. Giacomozzi, S. Colpo, B. Margesin, M. Bartek, "A General Purpose Reconfigurable MEMS-Based Attenuator for Radio Frequency and Microwave Applications", in Proc. of EUROCON, 2009, pp. 1201-1209

[2] J. lannacci , A. Faes, A. Repchankova , A. Tazzoli, G. Meneghesso, "An active heatbased restoring mechanism for improving the reliability of RF-MEMS switches", Journal of Microelectronics Reliability 51-9/11 (2011), pp.1869-1873.

[3] A. Tazzoli, J. lannacci, G. Meneghesso, " A positive exploitation of ESD events: Microwelding induction on ohmic MEMS contact.", in Proc. of the Electrical Overstress/Electrostatic Discharge Symp. 2011, pp 1-8.

[4] Papaioannou G, Papapolymerou J, Pons P and Plana R., "RF-MEMS dielectric charging: Dependence on dielectric film polarization procedures", in Proc. of AFRICON 2007, pp 1-6.

[5] T. Kuenzig, J. lannacci, G. Schrag, G. Wachutka, "Study of an active thermal recovery mechanism for an electrostatically actuated RFMEMS switch", in Proc. of Eurosime 2012, Portugal, 2012

[6] G. Schrag, R. Khaliliyulin, M. Niessner, and G. Wachutka, "Hierarchical Modeling Approach for Full-System Design and control of Microelectromechanical Systems," in Proc. of Eurosensors XXII, 2008, pp. 528-531

[7] G. Schrag, G. Wachutka, "System-Level Modeling of MEMS using Generalized Kirchhoffian Networks - Basic principles", in "System-level modeling of MEMS", Adv. Microand Nanosystems, Vol. 10, Wiley, 2013, pp. 19

[8] L. Gabbay, J. Mehner, and S. Senturia, "Computer-aided generation of reducedorder dynamic macromodels - I: Geometrically linear motion," J. Microelectromechanical Systems, vol. 9, 2000, pp. 262-269

[9] M. Niessner, G. Schrag, J. lannacci, G. Wachutka, "Macromodel-Based Simulation and Measurement of the Dynamic Pull-in of Viscously Damped RF-MEMS Switches", Sensors and Actuators A, Vol. 172 (1), 2011, pp. 269-279.

[10] G. Schrag , G. Wachutka, "Physically based modeling of squeeze film damping by mixedlevel system simulation," Sensors and Actuators A, vol. 97-98; 2002 , pp. 193-200.

[11] M.Niessner, J. lannacci, G. Schrag, "Mechanical Contact in System-level Models of Electrostatically Actuated RF-MEMS Switches: Experimental Analysis and Modeling", Proc. of Smart Sensors, Actuators, and MEMS (SPIE), 2011, Czech Republic, Vol. 8066, Bellingham, WA, USA, 2011, pp. 80660Y-1-9.

[12] M.Niessner, G.Schrag, J.lannacci, G.Wachutka, "Mixed-level modeling of squeeze film damping in MEMS: simulation and pressure-dependent experimental validation." Proc. of Transducers 2011, Beijing, China, pp. 1693. 